\title{
Investigación sobre la metodología pedagógica basada en el aula invertida durante la pandemia por la COVID-19: Un estudio cienciométrico
}

\author{
Estefanía Caballano-Infantes ${ }^{1}$, Daniel E. Di Zeo-Sánchez ${ }^{1}$, Pablo Sánchez-Núñez ${ }^{1}$ y Lorenzo G. \\ Borrego $^{2}$.
}

1. Universidad de Málaga, Campus Teatinos sn, 29071, Malaga, Spain. ecabinf@uma.es; danieldizeo@uma.es; psancheznunez@uma.es. ${ }^{2}$ Univ. Lyon, Université Claude Bernard Lyon 1, CNRS, INSA-Lyon, CPELyon, Institut de Chimie et Biochimie Moléculaires et Supramoléculaires, ICBMS, UMR 5246, Equipe CAtalyse, SYnthèse et ENvironnement (CASYEN), Campus LyonTech La Doua, Bâtiment Lederer,1 rue Victor Grignard, 69100 Villeurbanne, France.

\section{Abstract}

The flipped classroom is a pedagogical methodology focused on giving students an active role in the educational process, which aims to promote the development of key competencies. Recently, due to the COVID-19 pandemic, the flipped classroom has been on the rise, since it uses pedagogical strategies that allow learning to continue electronically. Despite this boom, the impact of this methodology has not yet been studied in the literature. Therefore, the objective of this study has been to provide a useful vision of the evolution of research on the flipped classroom during the pandemic, revealing the type of publications, the highly cited documents in this area, the publications that have served as a reference for the most relevant authors in this research field, the keywords, as well as the conceptual structure of this research field, through a bibliometric study.

This work offers an approach to the knowledge of the current state of development of research on the flipped classroom under the influence of the COVID-19 pandemic and can facilitate collaborations between researchers and the creation of new lines of research that help implement quality education.

Keywords: Flipped classroom; Coronavirus; SARS-CoV-2; Educational Innovation; Reporting; Scientometry; Bibliometrics; Scientific communication, Bibliometrix.

\section{Resumen}

El aula invertida es una metodología pedagógica centrada en conferir al alumnado un papel activo en el proceso educativo, que tiene como objetivo promover el desarrollo de competencias clave. Recientemente, debido a la pandemia por COVID-19, el aula invertida se ha visto en auge, ya que emplea estrategias pedagógicas que permiten continuar con el aprendizaje de forma telemática. A pesar de este auge aún no se ha estudiado el impacto de esta metodología en la literatura. Por ello, el objetivo de este estudio ha sido proporcionar una visión útil de la evolución de la investigación sobre el aula invertida durante la pandemia, revelando el tipo de publicaciones, los documentos altamente citados en esta 
área, las publicaciones que han servido de referencia para los autores más relevantes en este campo de investigación, las palabras clave, así como la estructura conceptual de este campo de investigación, a través de un estudio bibliométrico.

Este trabajo ofrece una aproximación al conocimiento del estado de desarrollo actual de la investigación sobre el aula invertida bajo la influencia de la pandemia por COVID-19 y puede facilitar la colaboraciones entre investigadores y la creación de nuevas líneas de investigación que ayuden a implementar la calidad educativa.

Palabras clave: Aula invertida; Coronavirus; SARS-CoV-2; Innovación Educativa; Informetría; Cienciometría; Bibliometría; Comunicación científica, Bibliometrix.

\section{Introducción}

Como si se tratara de poner la clase "patas para arriba", la pedagogía inversa o aula invertida, en inglés "Flipped Clasroom", propone educandos que intervienen en el aula con preguntas y desafíos por resolver. Es un método innovador que plantea una nueva fórmula en la que los estudiantes ejercen su trabajo en el temario fuera del centro, accediendo a los contenidos de interés para que, posteriormente, sea en el aula dónde se realicen actividades más participativas (razonar ideas, plantear hipótesis, iniciar debates, trabajos en conjunto, etc). Esta metodología constructivista precisa de unas pautas de actuación guiadas por un docente $[1,2]$.

En cuanto a la puesta en práctica de esta metodología pedagógica, recientemente la tutorización virtual se ha visto fomentada por la evolución de las TICs (Tecnologías de la Información y la Comunicación). El uso de recursos digitales beneficia la creación de grupos de colaboración para el aprendizaje en redes sociales y plataformas virtuales. Concretamente, se ha descrito que el uso de redes virtuales favorece la comunicación y el flujo de conocimiento, instaurando una atmósfera de colaboración idónea para mejorar el aprendizaje activo de los estudiantes. La tutorización del alumnado activa también promueve el intercambio de conocimientos e impresiones. Además, se observa que su uso amplía el interés y la motivación del alumnado optimizando el rendimiento académico[3].

Las estrategias basadas en el constructivismo estimulan el compromiso y la autonomía de cada individuo para construir su propio conocimiento, en definitiva, fomentan la competencia básica de aprender a aprender, entre otras [4].

Referente a las ventajas del aula invertida se destaca que esta metodología promueve un aprendizaje activo y significativo. Previamente, numerosos autores han descrito un efecto positivo en la participación y cooperación en el alumnado cuando cada persona individualmente interviene de forma activa en el proceso educativo[5], además de un impulso para el desarrollo de las habilidades cognitivas [7] y para la creación de un pensamiento crítico [6]. Además, el alumnado se habitúa a la adquisición de responsabilidades individuales y colectivas. Destacar, que esta metodología ayuda a consolidar el conocimiento, ya que el estudiantado cuenta con más tiempo para elaborar sus preguntas y resolver dudas [8]. En esta línea el aula 
invertida es una metodología que puede atender a la diversidad y ser inclusiva promoviendo así una enseñanza de calidad [9].

En paralelo al aula invertida, los esfuerzos por implementar la innovación docente han permitido desarrollar nuevas metodologías orientadas a impulsar el desarrollo de competencias clave entre las que destacan: el aprendizaje basado en proyecto [10], la realidad aumentada y/o la gamificación. En este sentido, estas metodologías han surgido como estrategias educativas destinadas a mejorar las habilidades cognitivas, así como las competencias científicas y digitales en el alumnado[11], resaltando su impacto positivo sobre la motivación y el rendimiento académico[12].

En la actualidad debido a la pandemia provocada por el coronavirus (SARS-CoV-2), se ha producido un auge en cuanto al empleo del aula invertida ya que permite continuar con el proceso de aprendizaje por vía telemática a través de plataformas virtuales, dónde el alumnado puede participar activamente a través de videollamadas y foros. En este sentido, diversos estudios han mostrado la efectividad de esta pedagogía durante la pandemia [13]. Concretamente se ha observado que el modelo combinado de enseñanza en línea con el aula invertida mejoró el aprendizaje, la atención y la evaluación de los estudiantes.

En cuanto a la aplicación de esta metodología durante la pandemia ha sido significativo el grado de absentismo y se han detectado problemas en la comunicación por vía telemática que plantean la necesidad de mejorar este modelo pedagógico instaurado de forma inminente tras declararse el estado de alerta sanitaria [14]. Por ello, más investigaciones sobre esta metodología y su aplicación son necesarias.

A pesar de que el aula invertida es una metodología pedagógica en auge durante la pandemia por COVID19, aún no se ha evaluado el impacto de esta enfermedad en las publicaciones sobre investigaciones relacionadas con esta metodología. En este trabajo se ha llevado a cabo un análisis cienciométrico con el objetivo de analizar los artículos más relevantes, las referencias más citadas, así como las palabras clave y la estructura conceptual de las investigaciones sobre el aula invertida durante la pandemia por COVID-19. $[15,16]$. Estos resultados pueden ayudar a los investigadores a ampliar sus conocimientos sobre el aula invertida y a establecer nuevas vías de colaboración orientadas a mejorar la calidad educativa no presencial. Además, conocer el estado actual de las investigaciones sobre el aula invertida durante la pandemia puede permitir seguir investigando y desarrollando diversos campos científicos, así como promover una mejora en la formulación de políticas de evaluación científica, investigación y financiación, en relación al establecimiento de acuerdos en materia de $\mathrm{I}+\mathrm{D}+\mathrm{i}$ y la creación de sinergias entre autores, países e instituciones.

\section{Objetivos}

Este trabajo pretende responder a las siguientes preguntas de investigación:

1. ¿Qué tipos de documentos han sido publicados relacionados relativo a la investigación sobre el aula invertida durante la pandemia por COVID-19?

2. ¿Cuáles son los documentos más citados globalmente sobre esta metodología pedagógica durante la pandemia por COVID-19? 
3. ¿Cuáles son las estructuras de conocimiento (estructura conceptual) sobre el aula invertida durante la pandemia?

\section{Desarrollo de la innovación}

Para la realización de este estudio bibliométrico se utilizó la base de datos Web of Science (WoS) como fuente para realizar el análisis cienciométrico. Web of Science es una plataforma propiedad de Clarivate Analytics, que consiste en una gran colección de bases de datos bibliográficas, citas y referencias de publicaciones científicas de cualquier disciplina del conocimiento, en ciencia, tecnología, ciencias sociales, artes y humanidades $[17,18]$.

La ecuación de búsqueda (Tabla 1) comprendió un conjunto de términos clave (título, resumen o palabras clave o "TITLE-ABSTRACT-KEYWORDS") relacionados con la pedagogía de aula invertida y el COVID-19 coronavirus (COVID-19) o SARS-CoV-2 durante el periodo 2020. No se estableció ningún criterio de exclusión relacionado con el idioma del documento publicado o el tipo de documento (actas de congresos, artículo o libro, etc.). Se obtuvieron un total de 36 documentos.

Tabla 1. Ecuación de búsqueda.

\begin{tabular}{|c|c|c|c|c|c|}
\hline Índices & Periodo & Query & & Resultados & $\begin{array}{l}\text { Fecha } \\
\text { descarga }\end{array}$ \\
\hline $\begin{array}{l}\text { SCI- } \\
\text { EXPANDED, } \\
\text { SSCI, A\&HCI, } \\
\text { CPCI-S, CPCI- } \\
\text { SSH, BKCI-S, } \\
\text { BKCI-SSH, } \\
\text { ESCI, CCR- } \\
\text { EXPANDED, } \\
\text { IC. }\end{array}$ & 2020 & \begin{tabular}{lc} 
TS=(("Flipped & \multicolumn{2}{c}{ Classroom") } \\
("COVID" & OR "COVID-19" \\
"COVID19" & OR "COVID 19" \\
"Coronavirus" & OR "SARS-CoV-2"))
\end{tabular} & $\begin{array}{r}\text { AND } \\
\text { OR } \\
\text { OR }\end{array}$ & 36 & 03.03 .3021 \\
\hline
\end{tabular}

Por otro lado, se ha empleado una herramienta de análisis cienciométrico debominada Bibliometrix. El paquete R Bibliometrix [19] es un conjunto de herramientas de código abierto para la investigación cuantitativa en cienciometría y bibliometría que incluye los principales métodos bibliométricos de análisis. Bibliometrix permite destacar los documentos, revistas, países, autores e instituciones más relevantes, así como estudiar y visualizar las distintas redes de colaboración en investigación, así como las tendencias establecidas o emergentes en un área específica del conocimiento. En este trabajo, los autores emplearon Bibliometrix con el fin de conocer los documentos más citados, las referencias locales más utilizadas, así como las palabras clave más recurrentes y por últimos las redes de concurrencia de palabras clave. 


\section{Resultados}

\section{Información del conjunto de datos}

En la Tabla 2 se muestra la información principal relacionada con el fichero de descarga de Web of Science en investigación en Flipped Classroom y COVID-19.

Tabla 2. Información del conjunto de datos.

\begin{tabular}{|l|l|}
\hline Descripción & Resultados \\
\hline $\begin{array}{l}\text { INFORMACIÓN PRINCIPAL SOBRE EL CONJUNTO DE } \\
\text { DATOS }\end{array}$ & \\
\hline Periodo & $2020: 2020$ \\
\hline Fuentes (Revistas, Libros, etc) & 27 \\
\hline Documentos & 36 \\
\hline Promedio de años desde la publicación & 1 \\
\hline Media de citas por documentos & 2,694 \\
\hline Promedio de citas al año por documento & 1,672 \\
\hline Referencias & 1150 \\
\hline TIPO DE DOCUMENTO & \\
\hline Artículo & 25 \\
\hline artículo; acceso temprano & 6 \\
\hline material editorial & 2 \\
\hline material editorial; acceso temprano & 1 \\
\hline Revisión & 2 \\
\hline CONTENIDO DEL DOCUMENTO & 0,383 \\
\hline Keywords Plus (ID) & 2,61 \\
\hline Palabras clave de autores (DE) & 138 \\
\hline AUTORES & 97 \\
\hline Autores & 11 \\
\hline Apariciones del autor & 83 \\
\hline Autores de documentos de autoría única & \\
\hline Autores de documentos con varios autores & \\
\hline AUTORÍA Y COLABORACIÓN & \\
\hline Documentos de autoría única & \\
\hline Documentos por autor & \\
\hline Autores por documento & \\
\hline
\end{tabular}


Investigación sobre la metodología pedagógica basada en el aula invertida durante la pandemia por la COVID-19: Un estudio cienciométrico

\begin{tabular}{|l|l|}
\hline Coautores por documentos & 2,69 \\
\hline Índice de colaboración & 3,32 \\
\hline
\end{tabular}

\section{Documentos relevantes: los documentos globalmente más citados}

En este trabajo, hemos evaluado los documentos más citados a nivel mundial para resaltar la relevancia de estas publicaciones y su impacto en la literatura. En la primera posición encontramos un trabajo titulado "Using Technology to Maintain the Education of Residents During the COVID-19 Pandemic" por Chick RC en 2020, con un total de 90 citas y 45 citas por año. En segundo lugar, encontramos un trabajo de Durfee SM, publicado en 2020 titulado "Medical Student Education Roadblock Due to COVID-19: Virtual Radiology Core Clerkship to the Rescue" con un total de 2 citas y una cita por año. El resto de las publicaciones que se detallan en esta lista han registrado un total de una cita y han sido descritos en la Tabla 3.

Tabla 3. Clasificación de los 5 trabajos más citados.

\begin{tabular}{|c|c|c|c|c|c|}
\hline Ranking & Publicación & DOI & Referencia & $\begin{array}{l}\text { Citaciones } \\
\text { totales }\end{array}$ & $\begin{array}{l}\text { Número } \\
\text { de } \\
\text { citaciones } \\
\text { por año }\end{array}$ \\
\hline 1. & CHICK RC, 2020, J SURG EDUC & 10.1016/j.jsurg.2020.03.018 & {$[20]$} & 90 & 45 \\
\hline 2. & $\begin{array}{llll}\text { DURFEE } & \text { SM, } & 2020, & \text { ACAD } \\
\text { RADIOL } & & & \\
\end{array}$ & 10.1016/j.acra.2020.07.020 & [21] & 2 & 1 \\
\hline 3. & $\begin{array}{l}\text { IZAGIRRE-OLAIZOLA J, 2020, } \\
\text { SUSTAINABILITY }\end{array}$ & $10.3390 / \mathrm{su} 122310137$ & {$[22]$} & 1 & 0,5 \\
\hline 4. & $\begin{array}{l}\text { PARKER EU, 2020, AM J CLIN } \\
\text { PATHOL }\end{array}$ & 10.1093/ajcp/aqaa154 & {$[23]$} & 1 & 0,5 \\
\hline 5. & LEE MW, 2020, J CHEM EDUC & 10.1021/j.jchemed.0c00881 & - & 1 & 0,5 \\
\hline
\end{tabular}

\section{Documentos más relevantes: referencias locales más recurrentes}

La Tabla 4 muestra la clasificación de las referencias más utilizadas por los autores de los artículos recuperados de Web of Science. Encontramos en primer lugar "Adopting Lightboard for a Chemistry Flipped Classroom to Improve Technology-Enhanced Videos for Better Learner Engagement" de Fung F, un trabajo que refleja las motivaciones y experiencia con el Lightboard, un interesante método de filmación 
usado en Flipped Classroom. El resto de trabajos de la clasificación acumulan el mismo número de citaciones (4).

Tabla 4. Clasificación de las 5 referencias más citadas.

\begin{tabular}{|c|c|c|c|}
\hline Ranking & Referencias citadas & Referencia & Citaciones \\
\hline 1. & $\begin{array}{l}\text { FUNG FM, 2017, J } \quad \text { CHEM } \\
\text { 10.1021/ACS.JCHEMED.7B00004 }\end{array}$ & {$[24]$} & 4 \\
\hline 2. & $\begin{array}{l}\text { HEW KF, 2018, BMC MED EDUC, V18, DOI 10.1186/S12909-018- } \\
1144-Z\end{array}$ & {$[25]$} & 4 \\
\hline 3. & $\begin{array}{llllllll}\text { SEERY MK, 2015, J } & \text { CHEM } & \text { EDUC, } & \text { V92, } & \text { P1566, } & \text { DOI } \\
\text { 10.1021/ED500919U } & & & & & & \end{array}$ & {$[26]$} & 4 \\
\hline 4. & $\begin{array}{llllll}\text { THAI NTT, 2017, } & \text { COMPUT } & \text { EDUC, } & \text { V107, } & \text { P113, } & \text { DOI } \\
\text { 10.1016/J.COMPEDU.2017.01.003 } & & & & \end{array}$ & {$[27]$} & 4 \\
\hline 5. & $\begin{array}{l}\text { ABEYSEKERA L, 2015, HIGH EDUC RES DEV, V34, P1, DOI } \\
10.1080 / 07294360.2014 .934336\end{array}$ & {$[28]$} & 3 \\
\hline
\end{tabular}

\section{Documentos más relevantes: nube de palabras}

En la Figura 1 se muestra la nube de palabras asociada a las Keyword Plus recuperadas en los trabajos de investigación. Encontramos que palabras como "compromiso", "rendimiento académico", "tecnología", "impacto" y "diseño futuro" suelen ser los conceptos más empleados para describir los trabajos de investigación.

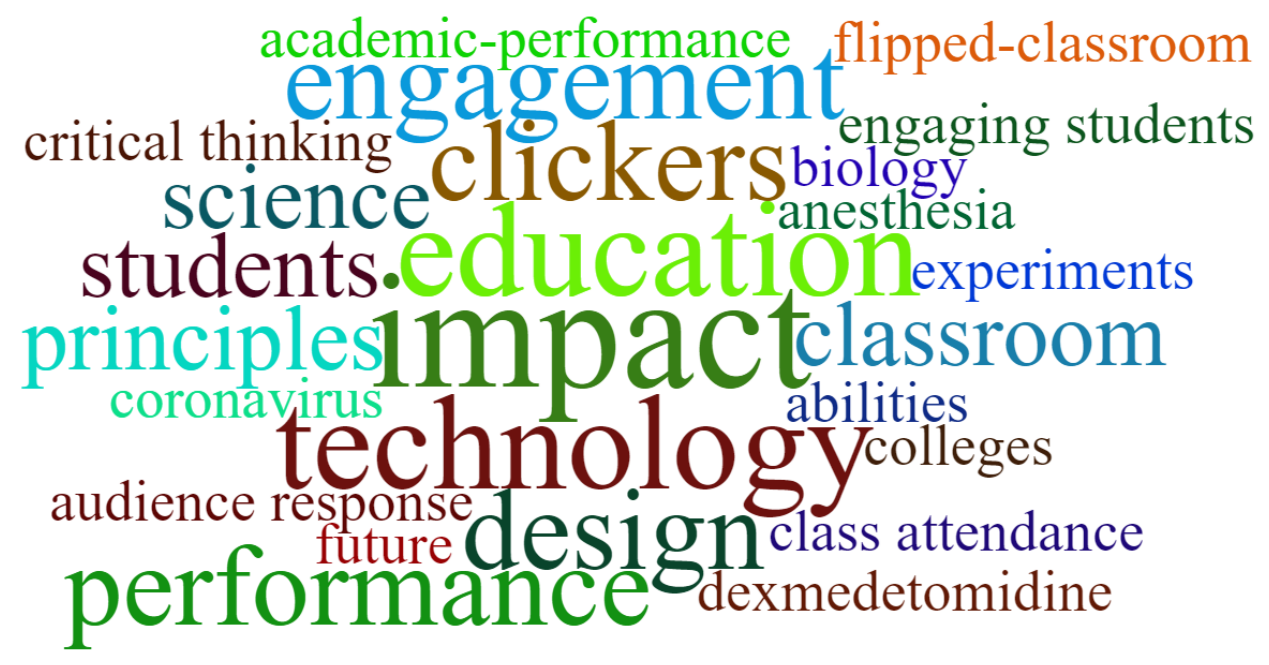

Figura 1. Nube de palabras. 


\section{Estructura conceptual: Red de coocurrencia de palabras clave}

Se identifican las relaciones entre los conceptos y las áreas temáticas principales dentro del estudio de Flipped Classroom y COVID-19. El análisis de co-palabras se entiende el estudio de las coocurrencias, o apariciones conjuntas, de dos términos en un texto dado con el propósito de identificar la estructura conceptual y temática de un dominio científico [29].

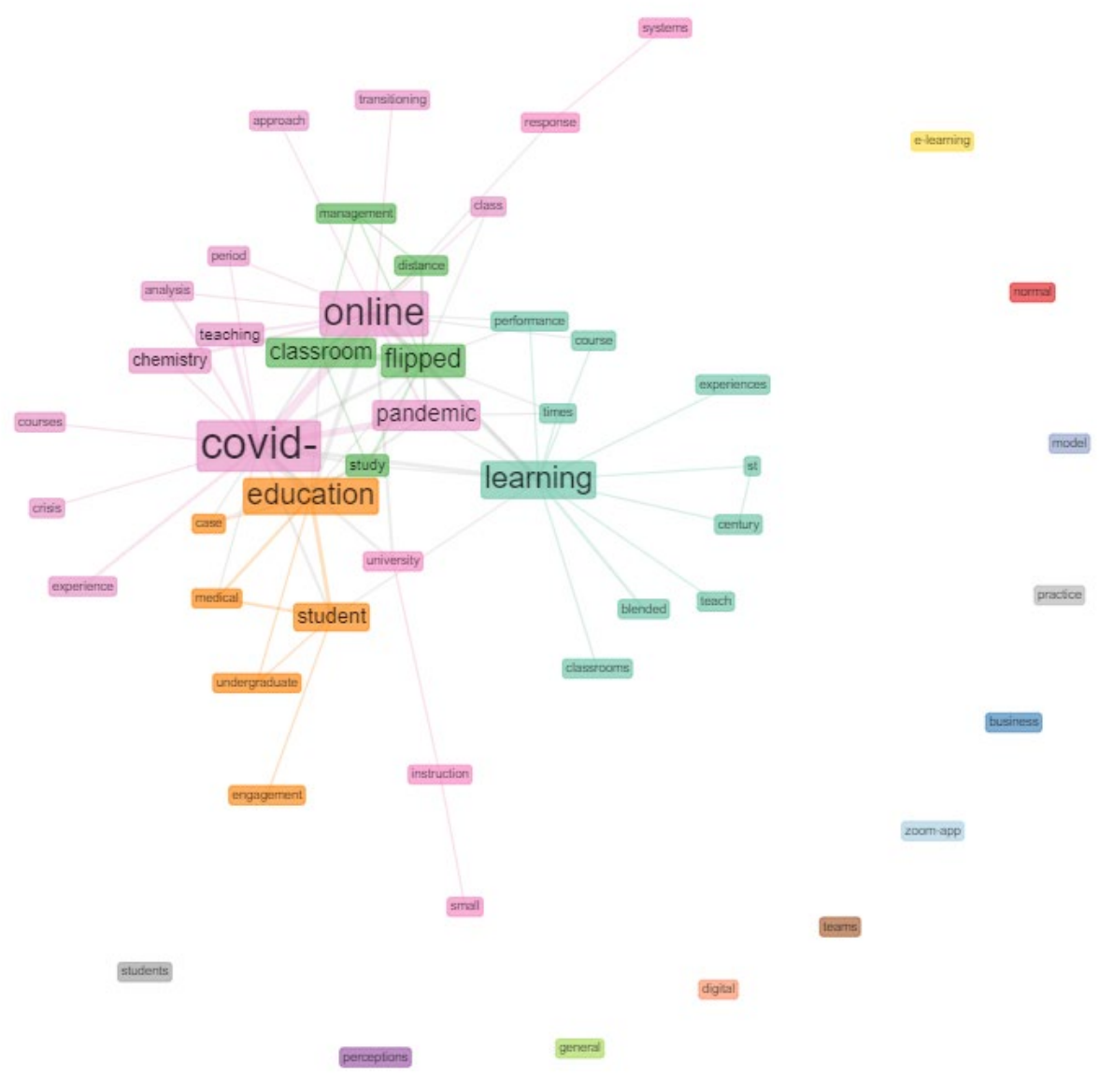

Figura 2. Red de co-ocurrencia. Las redes de co-ocurrencia son una interconexión compartida de términos basada en su presencia emparejada dentro de una unidad de texto definida. El tamaño de los términos indica su mayor o menor frecuencia de coincidencia con otro término en la red a lo largo de los HCP (coocurrencia). Parámetros de la red: campo (títulos), disposición de la red (Fruchterman \& Reingold), normalización (asociación), color de los nodos por año (No), algoritmo de agrupación (Louvain), número de nodos (5-50), eliminación de nodos aislados (No) y aristas mínimas (2). Parámetros gráficos: opacidad 
(0-0,7), número de etiquetas (0-50), cex de etiquetas (Sí), tamaño de las etiquetas (0-6), forma de los nodos (Box), tamaño de las aristas $(0,1-5)$ y aristas curvas (No).

En este trabajo hemos presentado el primer estudio bibliométrico sobre el impacto de la pandemia producida por el coronavirus SARS-COV-2 en los trabajos de investigación sobre la metodología pedagógica del aula invertida en 2020. Desde un punto de vista bibliométrico, previamente se ha estudiado la dinámica de la investigación en el aula invertida en la segunda década del siglo XXI. Además, en este trabajo Norliza Kushairi and Aidi Ahmi realizaron una predicción sobre la evolución de las investigaciones en el aula invertida en las próximas décadas a través de la ley de Lotka's [30].

En primer lugar, en este estudio hemos encontrado un total de 36 publicaciones relacionadas con estos ítems, de las cuales 25 han sido artículos, 2 han sido revisiones y el resto otro tipo de documentos descritos en la Tabla 2. Hemos detectado un total de 94 autores y destacamos que el $31 \%$ de estos trabajos han sido realizados y publicados por un solo autor.

En relación con estos trabajos hemos encontrado que el documento titulado "Using Technology to Maintain the Education of Residents During the COVID-19 Pandemic" por Chick RC en 2020, es la publicación más altamente citada con un total de 90 citas. En este trabajo Chick y colaboradores proponen varias soluciones innovadoras que incluyen el modelo de aula invertida, con actividades llevadas a cabo mediante teleconferencias, intervención de residentes en clínicas de telemedicina, simulación de procedimientos y el uso de videos quirúrgicos. En el campo de la cirugía, estos autores concluyen que a pesar de no haber sustituto para el aprendizaje práctico a través de la experiencia operativa y la atención directa al paciente, el aula invertida telemática puede ser una alternativa adecuada para mitigar la evolución en el aprendizaje de forma presencial durante la pandemia[20]. Este documento, al presentar un modelo de aprendizaje alternativo para un campo de trabajo tan estrictamente presencial, como es la cirugía, es de gran relevancia debido a que resulta extremadamente novedoso y útil mostrando una capacidad de flexibilidad y adaptación a las circunstancias actuales. Por ello, es de esperar que haya sido altamente citado y haya tenido un gran impacto en la literatura sobre los modelos pedagógicos telemáticos durante la pandemia por la COVID-19.

En cuanto a los documentos mayormente citados por los trabajos de aula invertida que hemos estudiado concretamente en este estudio, encontramos que investigaciones sobre innovación docente y aula invertida como el realizado por Fung FM y colaboradores en 2017[24] han sido los seleccionados. Concretamente, este estudio publicado en "Journal of Chemical Education" destaca los dos principales métodos para grabar videos y trabajar en un contexto de aula invertida. Por un lado, la pizarra clásica y por otro lado las presentaciones de PowerPoint, indicando que ambos carecen de interacción con el alumnado y requieren una gran inversión de tiempo en la edición a posteriori de los video educativos. En este sentido, el artículo presenta la pizarra digital como método alternativo para sustentar los problemas de las técnicas habituales. Esta tecnología novedosa, presentada por primera vez por el profesor Michael Peshkin en la Universidad Northwestern, podría ayudar a implicar a los estudiantes en el proceso educativo al facilitar una mayor conexión visual con el docente, logrando así unos de los principales objetivos didácticos. 
Por otro lado, entre los trabajos más altamente referenciado en los estudios sobre aula invertida durante la pandemia hemos encontrado el titulado "Flipped classroom frameworks improve efficacy in undergraduate practical courses - a quasi-randomized pilot study in otorhinolaryngology" por Tobias Dombrowski y colaboradores en 2018. Nuevamente se encuentra entre los documentos más relevantes sobre aula invertida una aplicación práctica de la misma en el campo de la medicina clínica quirúrgica. Además, se destaca una alta aceptación de esta metodología de aula invertida entre los estudiantes. Específicamente, estos autores muestran que el aprendizaje electrónico a través del aula invertida tiene un beneficio directo para adquirir conocimientos además de optimizar la experiencia práctica concretamente en el área de la otorrinolaringología [31].

De forma adicional, en los documentos más relevantes en este campo hemos analizado la red de palabras clave más significativas y entre ellas hemos subrayado: "Educación", "Tecnología", "Motivación", "Rendimiento académico", "Impacto", etc. En esta línea, el análisis de las palabras clave se ha descrito como esencial para que los investigadores busquen tendencias de investigación y conozcan el estado de desarrollo de un tema de interés [32]. En este sentido, hemos desvelado la estructura conceptual sobre la literatura enfocada en el impacto de la COVID-19 en la investigación sobre el aula invertida, encontrando una red de coocurrencia de términos interconectados dentro de un mismo documento. En esta línea, hemos encontrado 4 nodos bien diferenciados como son: "COVID-19", "educación en línea”, "aprendizaje” y "aula invertida"; lo cual indica la fuerte interrelación entre estos términos.

\section{Conclusiones}

El estudio cienciométrico presentado muestra por primera vez el impacto de la pandemia por COVID-19 en las investigaciones sobre la metodología pedagógica del aula invertida. En esta línea, en este trabajo hemos presentado la información principal procedente de la Web of Science relacionada con la investigación en aula invertida y COVID-19. Concretamente, se han presentado los trabajos mas relevantes en este campo, así como las referencia más citadas. Además, en este trabajo se han descrito datos de calidad de los trabajos publicados (número de citas y métricas de citas), las palabras clave reflejadas en las publicaciones, así como el mapa estructural (conceptos interrelacionados) de los datos recopilados de la base de datos Web of Science.

Por todo ello, este estudio puede ayudar a investigadores centrados en el aula invertida en el contexto social que vivimos actualmente a comprender el impacto de una pandemia sobre esta metodología, ya que permite conocer los trabajos más citados y los grupos de investigación involucrados, y podría servir de base para proponer vías para estudios posteriores y posibles colaboraciones internacionales que ayuden a mejorar la calidad educativa. Además, este trabajo muestra una metodología versátil para la realización de estudios bibliométricos, como es la herramienta Bibliometrix, que puede ser de utilidad para analizar el impacto de la pandemia en la evolución de la investigación sobre otras metodologías pedagógicas. En este sentido, el procedimiento bibliometrico presentado puede permitir conocer el estado de desarrollo de otras líneas de 
investigación docente de interés actual, como la gamificación o la realidad aumentada, orientadas a promover el aprendizaje flexible y adaptado a diversas circunstancias sanitarias, sociales y políticas.

\section{Referencias}

1. Sohrabi, B.; Iraj, H. Implementing flipped classroom using digital media: A comparison of two demographically different groups perceptions. Comput. Human Behav. 2016, 60, 514-524, doi:10.1016/j.chb.2016.02.056.

2. Bergmann, J.; Sams, A. Flip your classroom: Reach every student in every class every day. 2012.

3. Gómez, E. H., \& Vidal, F.M. Estrategias de aprendizaje basadas en entornos virtuales en educación secundaria. Revista científica electrónica de Educación y Comunicación en la Sociedad del Conocimiento, 15(2). 2015.

4. Lara Guerrero, J. Estrategias para un aprendizaje significativo-constructivista. Enseñanza Teach. Rev. Interuniv. didáctica 1997.

5. Ausubel, D.P. A cognitive theory of school learning. Psychol. Sch. 1969, 6, 331-335, doi:10.1002/15206807(196910)6:4<331::AID-PITS2310060402>3.0.CO;2-W.

6. Bonwell, C. C., \& Eison, J.A. Bonwell, C. C., \& Eison, J. A. (1991). Active Learning: Creating Excitement in the Classroom. 1991 ASHE-ERIC Higher Education Reports. ERIC Clearinghouse on Higher Education, The George Washington University, One Dupont Circle, Suite 630, Washington, DC 2; 1991;

7. Dewey, J. DEMOCRACY AND EDUCATION: AN INTRODUCTION TO THE PHILOSOPHY OF EDUCATION; 1916;

8. Fidalgo-Blanco, Á., Sein-Echaluce, M. L., \& García-Peñalvo, F.J. Fidalgo-Blanco, Á., Sein-Echaluce, M. L., \& García-Peñalvo, F. J. (2020). Ventajas reales en la aplicación del método de Aula InvertidaFlipped Classroom. Grupo GRIAL.; 2020;

9. Durán Gisbert, D.; Giné, C.G. La formación del profesorado para la educación enclusiva: Un proceso de desarrollo profesional y de mejora de los centros para atender la diversidad; Facultad de Ciencias de la Educación de la Universidad Central de Chile, 2018;

10. Llorens-Largo, F.; Villagrá-Arnedo, C.; Gallego-Durán, F.; Molina-Carmona, R. Campus Virtuales, 10(1), 2021; 2021; Vol. 10;.

11. De la Horra Villacé, I. Realidad aumentada, una revolución educativa. EDMETIC 2016, 6, 9, doi:10.21071/edmetic.v6i1.5762.

12. Subhash, S.; Cudney, E.A. Gamified learning in higher education: A systematic review of the literature. Comput. Human Behav. 2018, doi:10.1016/j.chb.2018.05.028.

13. Tang, T.; Abuhmaid, A.M.; Olaimat, M.; Oudat, D.M.; Aldhaeebi, M.; Bamanger, E. Efficiency of flipped classroom with online-based teaching under COVID-19. Interact. Learn. Environ. 2020, doi:10.1080/10494820.2020.1817761.

14. Durá, B.Z. Durá, B. Z. (2020). Deficiencias del aprendizaje autónomo en la educación telemática: Estudio de caso del periodo de confinamiento del COVID-19. In Tecnologías educativas y estrategias didácticas (pp. 1102-1111). Servicio de Publicaciones Universidad de M.

15. Fernández Cano, A.; Torralbo Rodríguez, M.; Rico, L.; Gutiérrez, P.; Maz, A. Scientometric analysis of Spanish doctoral dissertations on Mathematics Education (1976-1998). Rev. española Doc. Científica 2003, 26, 162-176, doi:10.3989/redc.2003.v26.i2.135. 
16. Jelvehgaran Esfahani, H.; Tavasoli, K.; Jabbarzadeh, A. Big data and social media: A scientometrics analysis. Int. J. Data Netw. Sci. 2019, 145-164, doi:10.5267/j.ijdns.2019.2.007.

17. Martínez, M.A.; Herrera, M.; Contreras, E.; Ruíz, A.; Herrera-Viedma, E. Characterizing highly cited papers in Social Work through H-Classics. Scientometrics 2015, 102, 1713-1729, doi:10.1007/s11192-014$1460-\mathrm{y}$.

18. Perez-Cabezas, V.; Ruiz-Molinero, C.; Carmona-Barrientos, I.; Herrera-Viedma, E.; Cobo, M.J.; Moral-Munoz, J.A. Highly cited papers in rheumatology: identification and conceptual analysis. Scientometrics 2018, 116, 555-568, doi:10.1007/s11192-018-2712-z.

19. Aria, M.; Cuccurullo, C. bibliometrix: An R-tool for comprehensive science mapping analysis. $J$. Informetr. 2017, 11, 959-975, doi:10.1016/j.joi.2017.08.007.

20. Chick, R.C.; Clifton, G.T.; Peace, K.M.; Propper, B.W.; Hale, D.F.; Alseidi, A.A.; Vreeland, T.J. Using Technology to Maintain the Education of Residents During the COVID-19 Pandemic. J. Surg. Educ. 2020, 77, 729-732, doi:10.1016/j.jsurg.2020.03.018.

21. Durfee, S.M.; Goldenson, R.P.; Gill, R.R.; Rincon, S.P.; Flower, E.; Avery, L.L. Medical Student Education Roadblock Due to COVID-19: Virtual Radiology Core Clerkship to the Rescue. Acad. Radiol. 2020, 27, 1461-1466, doi:10.1016/j.acra.2020.07.020.

22. Izagirre-Olaizola, J.; Morandeira-Arca, J. Business Management Teaching-Learning Processes in Times of Pandemic: Flipped Classroom at A Distance. Sustainability 2020, 12, 10137, doi:10.3390/su122310137.

23. Parker, E.U.; Chang, O.; Koch, L. Remote Anatomic Pathology Medical Student Education in Washington State. Am. J. Clin. Pathol. 2020, 154, 585-591, doi:10.1093/ajcp/aqaa154.

24. Fung, F.M. Adopting Lightboard for a Chemistry Flipped Classroom To Improve TechnologyEnhanced Videos for Better Learner Engagement. J. Chem. Educ. 2017, 94, 956-959, doi:10.1021/acs.jchemed.7b00004.

25. HEW, K.F.; LO, C.K. Flipped classroom improves student learning in health professions education: a meta-analysis. BMC Med. Educ. 2018, 18, 38, doi:10.1186/s12909-018-1144-z.

26. Seery, M.K. ConfChem Conference on Flipped Classroom: Student Engagement with Flipped Chemistry Lectures. J. Chem. Educ. 2015, 92, 1566-1567, doi:10.1021/ed500919u.

27. Thai, N.T.T.; De Wever, B.; Valcke, M. The impact of a flipped classroom design on learning performance in higher education: Looking for the best "blend" of lectures and guiding questions with feedback. Comput. Educ. 2017, 107, 113-126, doi:10.1016/j.compedu.2017.01.003.

28. Abeysekera, L.; Dawson, P. Motivation and cognitive load in the flipped classroom: definition, rationale and a call for research. High. Educ. Res. Dev. 2015, 34, 1-14, doi:10.1080/07294360.2014.934336.

29. GALVEZ, C. Análisis de co-palabras aplicado a los artículos muy citados en Biblioteconomía y Ciencias de la Información (2007-2017). Transinformação 2018, 30, 277-286, doi:10.1590/231808892018000300001.

30. Kushairi, N.; Ahmi, A. Flipped classroom in the second decade of the Millenia: a Bibliometrics analysis with Lotka's law. Educ. Inf. Technol. 2021, 1-31, doi:10.1007/s10639-021-10457-8.

31. Dombrowski, T.; Wrobel, C.; Dazert, S.; Volkenstein, S. Flipped classroom frameworks improve efficacy in undergraduate practical courses - a quasi-randomized pilot study in otorhinolaryngology. $B M C$ Med. Educ. 2018, 18, 294, doi:10.1186/s12909-018-1398-5.

32. Wen, H.; Huang, Y. Trends and performance of oxidative stress research from 1991 to 2010. Scientometrics 2012, 91, 51-63, doi:10.1007/s11192-011-0535-2. 